\title{
A Cut-Stem Inoculation Technique to Evaluate Soybean for Resistance to Macrophomina phaseolina
}

M. Twizeyimana, C. B. Hill, M. Pawlowski, and C. Paul, Department of Crop Sciences; and G. L. Hartman, United States Department of Agriculture-Agricultural Research Service and Department of Crop Sciences, University of Illinois, Urbana 61801

\begin{abstract}
Twizeyimana, M., Hill, C. B., Pawlowski, M., Paul, C., and Hartman, G. L. 2012. A cut-stem inoculation technique to evaluate soybean for resistance to Macrophomina phaseolina. Plant Dis. 96:1210-1215.

Charcoal rot of soybean is caused by the fungal pathogen Macrophomina phaseolina. Effective and reliable techniques to evaluate soybean for resistance to this fungus are needed to work toward a management scheme that would utilize host resistance. Three experiments were conducted to investigate the use of a cut-stem inoculation technique to evaluate soybean genotypes for resistance to $M$. phaseolina. The first experiment compared aggressiveness of $M$. phaseolina isolates collected from soybean on different soybean genotypes. Significant $(P<$ $0.05)$ differences among the isolates and genotypes for relative area under disease progress curve (RAUDPC) were found without a significant isolate-genotype interaction. The second experiment compared 14 soybean genotypes inoculated with $M$. phaseolina in multiple trials conducted in two environments, the greenhouse and growth chamber. Significant $(P<0.05)$ differences among environments and highly

significant $(P<0.001)$ differences among soybean genotypes for RAUDPC were found. The environment-genotype interaction was nonsignificant $(P>0.05)$. Soybean genotypes DT97-4290, DT987553, DT98-17554, and DT99-16864 had significantly $(P<0.05)$ lower RAUDPC than 7 of the 14 genotypes. The third experiment evaluated resistance in selected Phaseolus spp. and soybean genotypes. The range of RAUDPC for Phaseolus spp. was similar to that of soybean. The Phaseolus lunatus 'Bush Baby Lima' had significantly $(P<$ $0.05)$ lower RAUDPC than $P$. vulgaris genotypes evaluated. The cutstem inoculation technique, which has several advantages over field tests, successfully distinguished differences in aggressiveness among $M$. phaseolina isolates and relative differences among soybean genotypes for resistance to $M$. phaseolina comparable with results of field tests.
\end{abstract}

Charcoal rot of soybean (Glycine max (L.) Merr.) is caused by Macrophomina phaseolina (Tassi) Goid., a soilborne pathogen reported to infect over 500 cultivated and wild plant species, including economically important field crops such as common bean (32), sorghum (9), soybean (22,31), and sunflower (14). Estimated annual losses in soybean of 0.68 million metric tons were attributed to charcoal rot from 1989 to 1991 in the North Central Region of the United States (8), while 0.71, 0.84, and 0.6 million metric tons were estimated annual losses from 2006, 2007, and 2008, respectively, in the entire United States (15).

In the field, infection of $M$. phaseolina originates from microsclerotia in the soil or from conidia, produced on infected plant tissues or residue, that are splashed onto host plant parts during rain events (6). All plant parts of soybean are susceptible to infection. $M$. phaseolina hyphae initially grow intercellularly and travel through the vascular cylinder of the plant. Infection often begins with a biotrophic phase with no visible symptoms but changes in environmental conditions, host plant stress, or plant maturation may promote a necrotrophic phase. This phase is characterized by visible plant damage, such as plant wilting and flagging of branches, resulting from the mechanical plugging of vascular tissue by fungal parts, necrosis caused by phytotoxins, enzymatic action, and mechanical pressure during cell wall penetration (31). Severe wilting in soybean occurs mostly during hot, dry weather accompanied by conditions that reduce plant vigor, such as poor soil fertility and high seeding rates. Other symptoms include blight of emerging seedlings, early maturation, and incomplete pod filling (31). A combination of these symptoms usually occurs in severe cases of the disease in soybean, which can result in significant yield losses.

Corresponding author: G. L. Hartman, E-mail: ghartman@illinois.edu

Accepted for publication 14 March 2012.

http://dx.doi.org/10.1094/PDIS-02-12-0126-RE

(C) 2012 The American Phytopathological Society
Current strategies available for the management of charcoal rot in soybean mainly involve the use of cultural methods such as staggering of planting dates, crop rotation, reducing plant densities, and irrigation $(2,11,12,36)$. These methods have not been effective or widely adopted by soybean growers; however, other control methods, including fungicide applications to seed and soil (24) and biological control using hyperparasitism $(27,29)$, have given limited disease control in soybean as well as in other crops. Management through host resistance also has been promoted $(2,3,20,21,26,30)$. It was reported that soybean genotypes with the lowest levels of $M$. phaseolina root colonization produce the highest yields $(26,28)$. In 2006, the breeding line DT97-4290 was registered for its high yield and moderate resistance to $M$. phaseolina (25); however, soybean cultivars with high levels of resistance to $M$. phaseolina are not commercially available.

Finding new sources of resistance to plant pathogens requires reliable disease evaluation techniques. To date, most studies evaluating soybean germplasm for resistance to M. phaseolina have used field plots, either inoculated with the fungus or chosen for their history of the disease and relying on the presence of field inoculum $(2,3,20,21,26,30)$. As with most field experiments, variability among fields with different soil characteristics and microflora that can interact with M. phaseolina (39) or across locations and seasons with varying weather patterns and moisture, can produce inconsistent results between field experiments. Field tests that rely on field inoculum are subject to reduced precision in detecting true differences among genotypes because of nonuniform inoculum concentration and nonrandom inoculum distribution within and between fields (23). Unfortunately, proven techniques to inoculate plants in the field to control variability in inoculum among test plots have not yet been established. An issue specifically for the field evaluation of soybean genotypes is the effect of plant maturity on the relative timing of symptom development. Soybean genotypes with different maturity will not reach growth stage R7 (10), the stage in which evaluations are usually performed $(20,21,30)$, at the same date under identical environmental conditions $(26,36)$, often producing confounding results. Moreover, in most locations, 
only a single field experiment is completed per season. Such limitations inherent in field experiments may have hindered progress toward identifying new charcoal rot resistance sources and breeding for such resistance in soybean. Non-field testing under semicontrolled conditions in a greenhouse or in controlled conditions in plant growth chambers using standardized environmental conditions and inoculation techniques may compliment or help to overcome the limitations of field-testing.

Soybean genotypes have been screened for resistance to charcoal rot under controlled environments in the greenhouse using full-grown soybean plants (35) and in growth chambers using seedlings (3); however, such screening has not been widely used, possibly because of the lack of a reliable technique. In recent years, our laboratory developed and applied a cut-stem technique to inoculate soybean with Phomopsis longicolla and Sclerotinia sclerotiorum $(16,38)$. This technique has been effective in identifying partial resistance to these two diseases and has proven crucial in phenotyping populations segregating for resistance to map quantitative trait loci controlling resistance to Sclerotinia stem rot. In addition to investigating the effectiveness of this technique for screening soybean for resistance to M. phaseolina, we wanted to know if it was applicable to other crop plants with plant architecture similar to that of soybean, such as Phaseolus spp., which also has been evaluated under controlled conditions $(5,13)$ but not using the cutstem inoculation technique. The objectives of this study were to evaluate the use of the cut-stem inoculation technique in controlled conditions to (i) compare the aggressiveness of different $M$. phaseolina isolates and (ii) screen for resistance to charcoal rot among selected soybean and Phaseolus spp. genotypes.

\section{Materials and Methods}

Plant material and planting. Sixteen soybean genotypes and six genotypes of Phaseolus spp. were used in this study. Most of the soybean genotypes were selected from a panel of genotypes that were previously tested for charcoal rot resistance in the field (21). Lines with the DT prefix were obtained from the United States Department of Agriculture-Agricultural Research Service, Stoneville, MS; lines with the LS prefix were obtained from Southern Illinois University, Carbondale; and 'Croton', 'Pana', 'Pharaoh', and 'Spencer' were public releases from universities in the Midwest. The four Phaseolus vulgaris plant introduction (PI) accessions were selected and obtained from the National Plant Germplasm System based on their previously reported (37) resistance to ashy stem blight caused by $M$. phaseolina. 'Bush Baby Lima' and 'Bush Yellow Wax' bean were purchased locally.

Experiments were conducted in a greenhouse or a growth chamber. Seed was over-sown in 48-pot plastic inserts (number 1204; Hummert International) and thinned to one plant per pot after plant emergence. Each insert, with 12 rows of four pots, was filled with soil-less mix (Sunshine Mix, LC1; Sun Gro Horticulture Inc.), placed inside a flat with drainage holes (number T1020; Hummert International), and fertilized at planting with slow-release pellets (Osmocote 19-6-12; 1 to 2 pellets $/ \mathrm{cm}^{2}$ ). Depending on the experiment and trial within the experiment, flats were placed in a growth chamber (Percival Scientific, Inc.) maintained at $30^{\circ} \mathrm{C}$ and a $16-\mathrm{h}$ photoperiod with a light intensity approximately $500 \mu \mathrm{mol} \mathrm{m} \mathrm{m}^{-2} \mathrm{~s}^{-1}$ or on a bench inside a greenhouse at $30 \pm 2{ }^{\circ} \mathrm{C}$, with supplemental illumination provided by $500-\mathrm{W}$ high-pressure sodium vapor lamps.

Isolate aggressiveness experiment. Two trials, using the cutstem inoculation technique as described below, were conducted using four isolates of M. phaseolina; namely Conway, Kibler, and Pinetree (collected in Arkansas and obtained from Dr. John Rupe, University of Arkansas, Fayetteville), and Mp3, a local isolate collected in east-central Illinois. Each isolate was cultured on potato dextrose agar (Difco Laboratories) and maintained in an incubator at $28^{\circ} \mathrm{C}$. Trial 1 used moderately resistant DT97-4290 (25); moderately susceptible LS98-1430; susceptible LS94-3207 (21); Spencer, which had shown partial resistance in our preliminary experiments; and two genotypes, Pana and LS98-0358, that had no previous reported evaluations. Trial 2 used DT97-4290, Spencer, and the susceptible Pharaoh (21). The isolate aggressiveness experiment trials were conducted as factorial experiments. Factors (isolates and soybean genotypes) were arranged in a randomized complete block design with three blocks. The experimental unit in both trials of this experiment was the row of four plants of each isolate-genotype factor combination in each block. Trial 1 was contained in six flats with two flats per block and trial 2 was contained in three flats with a single flat for each block. Both trials were conducted in growth chambers under conditions previously described.

Experiment to evaluate resistance to $M$. phaseolina in soybean genotypes. Fourteen soybean genotypes were evaluated for M. phaseolina resistance using the cut-stem technique. Of the 14 genotypes, 9 were selected based on the results of a field study (21) in order to have a panel of genotypes that exhibited a range of field responses to $M$. phaseolina for comparison with the results of the non-field evaluation technique. There were three trials conducted in the growth chamber and two trials conducted in the greenhouse under the environmental conditions previously described. The experimental design for all five trials in this experiment was a randomized complete block design with three blocks and four plants (experimental units) per block. Each trial was contained in six flats with two flats per block. Plants in two of the growth chamber trials were inoculated with $M$. phaseolina isolate $\mathrm{Mp} 3$; the Pinetree isolate was used in the third growth chamber trial and the two greenhouse trials.

Experiment to evaluate resistance to $M$. phaseolina in genotypes of Phaseolus spp. To determine whether the cut-stem inoculation technique was suitable for evaluating resistance to $M$. phaseolina in another crop, six genotypes consisting of two Phaseolus spp. and four P. vulgaris common bean accessions were obtained from the National Plant Germplasm System and selected because they were listed as having resistance to ashy stem blight caused by M. phaseolina (37). Two commercial bean genotypes obtained locally, P. lunatus Bush Baby Lima and P. vulgaris Bush Yellow Wax, were also included in the experiment along with three soybean genotypes: DT97-4290, Pharaoh, and Spencer. This experiment, which was repeated once with a different randomization of genotypes under previously described greenhouse conditions, was arranged in a randomized complete block design with three blocks and four plants (experimental units) per block. The experiment was contained in three flats with a single flat per block. The Pinetree isolate was used for inoculation.

Cut-stem inoculation technique. The stem apex of each sixweek-old V2-stage (10) soybean plant was cut $25 \mathrm{~mm}$ above the unifoliolate node with a sharp razor blade. The open end of a 10- to $200-\mu$ l pipette tip (Fisher Scientific) was pushed into the margin of an actively growing $M$. phaseolina culture growing on potato dextrose agar, and a circular disk of fungal mycelium and agar was cut and removed. The pipette tip containing the agar disk with $M$. phaseolina mycelium was immediately placed over the cut stem and pushed down as far as possible in order to embed the stem into the medium and to secure the tip onto the stem. Phaseolus spp. genotypes were also inoculated in this manner.

Measurement of necrosis caused by $\boldsymbol{M}$. phaseolina. Three days after inoculation, the pipette tips were removed from each plant and discarded. Linear stem necrosis (in millimeters) caused by $M$. phaseolina infection was measured on each plant with a ruler and recorded every 3 days until the end of each trial 13 to 15 days post inoculation. Because the $M$. phaseolina infection often caused part of the stem apex to rot and fall off, eliminating a portion of the top of the plant, measurements were taken from the unifoliolate node. If the edge of the necrosis did not reach down to the unifoliolate node, the distance from the node up to the lower edge of the necrosis was taken and this distance then subtracted from $25 \mathrm{~mm}$, which was the distance between the unifoliolate node and the inoculation point, to obtain the length of necrosis. If the necrosis progressed below the unifoliolate node, the distance between the unifoliolate node down to the lowest part of the necrosis 
was measured and this distance then added to $25 \mathrm{~mm}$ to obtain the length of necrosis.

Data analysis. The linear extent of stem necrosis ( $\mathrm{mm})$, caused by $M$. phaseolina infection that developed from the inoculation point at the cut-stem apex and measured on each inoculated plant over time, was used to calculate the area under the disease progress curve (AUDPC; 4). The mean AUDPC of the four plants in each row was the experimental unit. Due to differences in number of days when necrosis measurements were recorded in the experiments, the relative area under the disease progress curve (RAUDPC; 4,18) was calculated to standardize the AUDPC data. This was done by dividing the AUDPC values by the number of days between the first and last date of necrosis measurement. Prior to statistical analyses, RAUDPC data were log-transformed to normalize the data and correct for non-constant variance among samples.

In the isolate aggressiveness experiment, the effects of isolate and genotype on the RAUDPC were determined using restricted or residual maximum likelihood (REML) analysis (JMP, version 9.03, 2010; SAS Institute Inc.). Blocks nested within each trial were random effects. Each trial was analyzed separately because different soybean genotypes were used. To minimize type I errors, means were separated using Fisher's protected least significant difference (LSD) experiment at $\alpha=0.05$ only when analysis of variance indicated significant differences $(P<0.05)$ among isolates or genotypes.

For the experiment to evaluate resistance to M. phaseolina in soybean genotypes, the effects of environment and genotype and their interaction on RAUDPC was determined using REML analysis as previously described. Environments and genotypes were fixed effects. Trials and blocks were random effects. Trials were nested within environments and blocks were nested within trials. Means were separated using Fisher's LSD.

Significance of variance heterogeneity among repeats of the experiment to evaluate $M$. phaseolina resistance in Phaseolus spp.

Table 1. Restricted or residual maximum likelihood analyses for relative area under the disease progress curve (RAUDPC) among four Macrophomina phaseolina isolates in two trials with different soybean (Glycine max) genotypes following cut-stem inoculation technique ${ }^{\mathrm{z}}$

\begin{tabular}{|c|c|c|c|c|}
\hline \multirow[b]{2}{*}{ Source of variation } & \multicolumn{2}{|c|}{ Trial 1} & \multicolumn{2}{|c|}{ Trial 2} \\
\hline & df & $F$ ratio & df & $F$ ratio \\
\hline Isolate & 3 & $23.4 * * *$ & 3 & $7.8 * * *$ \\
\hline Genotype & 5 & $6.3 * * *$ & 2 & $3.8^{*}$ \\
\hline Isolate $\times$ genotype & 15 & 0.5 & 6 & 0.6 \\
\hline
\end{tabular}

${ }^{\mathrm{z}}$ Isolates and genotypes were fixed effects. Blocks were random effects nested within trials. Mean RAUDPC for each four-row experimental unit was log-transformed prior to analysis to normalize the data and correct for non-constant variance; $*$ and $* * *$ indicated significant differences were found at $P=0.05$ and 0.001 , respectively.

Table 2. Relative area under disease progress curve (RAUDPC) of infection by four Macrophomina phaseolina isolates, collected from soybean, on selected soybean (Glycine max) genotypes using a cut-stem inoculation technique ${ }^{\mathrm{y}}$

\begin{tabular}{lll}
\hline Isolate $^{\mathbf{z}}$ & Trial 1 & Trial 2 \\
\hline Pinetree & $42.4 \mathrm{a}$ & $67.6 \mathrm{a}$ \\
Mp3 & $30.0 \mathrm{~b}$ & $63.8 \mathrm{a}$ \\
Conway & $26.0 \mathrm{~b}$ & $50.6 \mathrm{~b}$ \\
Kibler & $14.2 \mathrm{c}$ & $45.0 \mathrm{~b}$ \\
Mean & 25.7 & 56.2 \\
\hline
\end{tabular}

${ }^{y}$ RAUDPC was calculated by dividing the AUDPC (area under disease progress curve) values by the number of days between the first and last date of necrosis measurement. Means followed by a common letter were not significantly different by least significant difference $(P<0.05)$. Coefficient of variation was 18.7 and $6.6 \%$ for trial 1 and 2 , respectively.

${ }^{\mathrm{z}} \mathrm{Mp} 3$ isolate was a local isolate from Illinois collected in 1998; Conway, Kibler, and Pinetree were collected by J. Rupe (University of Arkansas, Fayetteville). was tested using Bartlett's test (JMP, version 9.03, 2010; SAS Institute Inc.) in order to determine whether data from the repeats could be pooled for combined analysis. The effect of genotype on RAUDPC was determined using REML and means were separated using Fisher's LSD. Genotypes were fixed effects and blocks were random effects.

\section{Results}

Isolate aggressiveness experiment. There were significant $(P<$ $0.05)$ differences among soybean genotypes and isolates without significant $(P>0.05)$ isolate-genotype interaction for RAUDPC in both trials (Table 1), indicating that the soybean genotypes had different levels of resistance to $M$. phaseolina infection and that the $M$. phaseolina isolates were different for aggressiveness. The Pinetree isolate produced significantly $(P<0.05)$ higher RAUDPC than the other three isolates in trial 1, and significantly higher RAUDPC than isolates Conway and Kibler in trial 2 (Table 2). LSD separated the soybean genotypes into two classes in trial 1, with the four genotypes Spencer, LS98-1430, DT97-4290, and Pana having significantly $(P<$ 0.05) lower RAUDPC than LS98-0358 and LS94-3207 (Table 3). DT97-290 had significantly $(P<0.05)$ lower RAUDPC than Spencer and Pharoah in trial 2 (Table 3). The lack of isolate-genotype interaction indicated that relative differences in isolate aggressiveness were consistent on each soybean genotype. Trial 2 had a higher mean RAUDPC (56.2) and lower coefficient of variation (CV; 6.6\%) than trial 1, with a mean RAUDPC of 25.7 and a CV of $18.7 \%$

Experiment to evaluate resistance to $M$. phaseolina in soybean genotypes. Because there was no significant interaction between genotypes and isolates in the aggressiveness experiment, the effect of using either the Mp3 or the Pinetree isolate in this experiment was not determined. All soybean genotypes developed stem necrosis during the course of the experiments, indicating that there was no immunity or complete resistance to $M$. phaseolina.

There were significant $(P<0.05)$ differences between the growth chamber and greenhouse trials and highly significant $(P<$ 0.01 ) differences among the 14 soybean genotypes for RAUDPC, without significant $(P>0.05)$ interaction between the two factors, (Table 4). The mean RAUDPC in greenhouse trials was 39.9, which was significantly higher $(P<0.05)$ than the mean of 20.5 in the growth chamber trials. The $\mathrm{CV}$ for the greenhouse trials was $9.8 \%$ whereas, for the growth chamber trials, it was $13.5 \%$. Genotypes DT97-4290, DT98-7553, DT99-17554, and DT9916864 had significantly lower $(P<0.05)$ RAUDPC than 7 of the

Table 3. Relative area under the disease progress curve (RAUDPC) for Macrophomina phaseolina infection on selected soybean (Glycine max) genotypes using a cut-stem inoculation technique in two trials ${ }^{\mathrm{y}}$

\begin{tabular}{ll}
\hline Genotype $^{\mathbf{z}}$ & RAUDPC \\
\hline Trial 1 & \\
Spencer & $18.9 \mathrm{a}$ \\
LS98-1430 & $21.6 \mathrm{a}$ \\
DT97-4290 & $22.0 \mathrm{a}$ \\
Pana & $25.3 \mathrm{a}$ \\
LS98-0358 & $36.5 \mathrm{~b}$ \\
LS94-3207 & $38.6 \mathrm{~b}$ \\
Mean & 25.7 \\
Trial 2 & \\
DT97-4290 & $49.1 \mathrm{a}$ \\
Spencer & $58.5 \mathrm{~b}$ \\
Pharoah & $61.1 \mathrm{~b}$ \\
Mean & 56.2 \\
\hline
\end{tabular}

y RAUDPC was calculated by dividing the area under disease progress curve values by the number of days between the first and last date of necrosis measurement. Means followed by a common letter were not significantly different by the least significant difference at $P<0.05$. Coefficient of variation was 18.7 and $6.6 \%$ for trial 1 and 2 , respectively.

${ }^{z}$ Genotypes with DT prefix were obtained from the United States Department of Agriculture-Agricultural Research Service, Stoneville, MS; lines with LS prefix were obtained from Southern Illinois University, Carbondale, IL; and the remaining genotypes were public releases from universities in the Midwest. 
14 genotypes (Table 5). LS94-3207 had significantly higher $(P<$ 0.05) RAUDPC than 12 genotypes.

Experiment to evaluate resistance to $M$. phaseolina in genotypes of Phaseolus spp. Bartlett's test $(F$ ratio $=1.2 ; P=0.3$ ) indicated that variance for RAUDPC in the first and repeated trial was not significantly different. Therefore, the data from both trials were pooled for combined analysis. Highly significant differences $(F$ ratio $=7.9 ; P=0.006)$ were observed among six Phaseolus and three soybean genotypes for RAUDPC. Bush Baby Lima ( $P$. lunatus $)$ had a significantly lower $(P<0.05)$ RAUDPC than all of the $P$. vulgaris genotypes and G. max genotypes Spencer and Pharoah but not significantly different $(P>0.05)$ from the soybean genotype DT97-4290 (Table 6). RAUDPC among the $P$. vulgaris genotypes was not significantly different $(P>0.05)$ from each other or from Pharoah. RAUDPC on DT97-4290 was significantly lower $(P<$ $0.05)$ than RAUDPC on all of the $P$. vulgaris genotypes. The mean RAUDPC was 50.1 and CV was $12.2 \%$ for this experiment.

\section{Discussion}

The relative differences among nine soybean genotypes found using the cut-stem technique were in agreement with

Table 4. Restricted or residual maximum likelihood analysis for relative area under the disease progress curve (RAUDPC) among 14 soybean (Glycine max) genotypes and between environments (growth chamber and greenhouse) and environment-genotype interaction following inoculation with Macrophomina phaseolina using a cut-stem inoculation technique

\begin{tabular}{lcc}
\hline Source of variation & df & $\boldsymbol{F}$ ratio $^{\mathbf{z}}$ \\
\hline Environment & 1 & $25.5^{*}$ \\
Genotype & 13 & $6.8^{* * *}$ \\
Environment $\times$ genotype & 13 & 0.6 \\
\hline
\end{tabular}

${ }^{y}$ Environments and genotypes were fixed effects. Trials and blocks were random effects. Trials were nested within environments and blocks were nested within trials. There were three trials conducted in the growth chamber and two trials conducted in the greenhouse. Mean RAUDPC for each four-row experimental unit was log-transformed prior to analysis to correct for non-normality.

z Asterisks: * and *** indicate that significant differences were found at $P$ 0.05 and 0.001 , respectively.

Table 5. Relative area under the disease progress curve (RAUDPC) for Macrophomina phaseolina infection on 14 soybean (Glycine max) genotypes using a cut-stem inoculation technique in five trials (three from growth chamber and two from the greenhouse)

\begin{tabular}{lc}
\hline Genotype $^{\mathbf{y}}$ & RAUDPC $^{\mathbf{z}}$ \\
\hline DT97-4290 & $21.6 \mathrm{a}$ \\
DT98-7553 & $21.7 \mathrm{a}$ \\
DT99-17554 & $22.8 \mathrm{a}$ \\
DT99-16864 & $23.7 \mathrm{a}$ \\
Spencer & $23.9 \mathrm{ab}$ \\
LS98-3257 & $26.5 \mathrm{abc}$ \\
LS98-2574 & $26.8 \mathrm{abc}$ \\
LS98-0719 & $29.9 \mathrm{bcd}$ \\
LS98-1430 & $30.1 \mathrm{~cd}$ \\
DT99-17483 & $31.1 \mathrm{~cd}$ \\
Croton & $32.1 \mathrm{~cd}$ \\
LS92-1088 & $33.8 \mathrm{~d}$ \\
Pharoah & $36.8 \mathrm{de}$ \\
LS94-3207 & $42.7 \mathrm{e}$ \\
\hline
\end{tabular}

${ }^{y}$ Genotypes with DT prefix were obtained from the United States Department of Agriculture-Agricultural Research Service, Stoneville, MS; genotypes with LS prefix were obtained from Southern Illinois University, Carbondale, IL; and the remaining genotypes were public releases from universities in the Midwest.

${ }^{\mathrm{z}}$ RAUDPC was calculated by dividing the area under disease progress curve by the number of days between the first and last date of necrosis measurement. Means followed by common letters were not significantly different by the least significant difference test at $P=0.05$. The mean RAUDPC in the greenhouse trials was 38.9 , which was significantly higher $(P>0.05)$ than the mean of 20.5 in the growth chamber trials. The CV was 9.8 and $13.5 \%$, respectively, for greenhouse and growth chamber trials.
CFU index (CFUI) results for the same nine genotypes evaluated in the field (21). Low levels of necrosis on DT974290, DT98-7553, DT99-16864, and DT99-17554 were comparable with low CFUIs recorded on these genotypes in field evaluations (21). This result confirms that these genotypes are excellent sources of charcoal rot resistance in soybean. Consistent with other research results (field or greenhouse evaluations; 3,26), complete resistance was not found among the soybean genotypes tested in this study.

There are several advantages to using the non-field cut-stem inoculation technique compared with field-testing soybean for resistance to $M$. phaseolina. A uniform amount of inoculum is placed directly onto an identical infection court on each plant using the cut-stem inoculation technique, which minimizes potential infection escapes, whereas plants in field tests are often indirectly inoculated by placing inoculum in the furrow with the seed at planting (21). The cut-stem inoculation technique could be further improved with the use of M. phaseolina conidial inoculum (17), enabling precise quantitative inoculation which would help reduce experimental error and improve precision in evaluating $M$. phaseolina resistance. Level of disease caused by M. phaseolina on each plant is directly quantified using the cut-stem technique by measuring the extent of necrosis caused by the pathogen, significantly improving the precision of the resistance evaluation over indirect disease measurements, such as the CFUI method (21), used in field tests. Each cycle of evaluation using the cut-stem technique can be completed in about 8 weeks (6 weeks for plant growth and 2 weeks for disease evaluation) compared with a full season required to complete a field test, making the cut-stem technique suitable to high-throughput screening of germplasm or phenotyping genetic mapping populations for $M$. phaseolina resistance. Most importantly, environmental variation and plant maturity differences among soybean genotypes which can contribute to inconsistent results among field tests can be controlled using the non-field cut-stem technique in semi-controlled greenhouse or controlled growth chamber environments, further reducing experimental error and improving reliability of results across $M$. phaseolina resistance evaluations.

The lack of environment-genotype interaction found in this study indicated that relative differences between soybean genotypes for $M$. phaseolina resistance were consistent in both greenhouse and growth chamber trials, although RAUDPC was higher

Table 6. Relative area under the disease progress curve (RAUDPC) among six Phaseolus spp. and three soybean (Glycine max) genotypes inoculated with Macrophomina phaseolina using a cut-stem inoculation technique in two greenhouse trials

\begin{tabular}{ll}
\hline Genotype $^{\mathbf{y}}$ & RAUDPC $^{\mathbf{z}}$ \\
\hline Phaseolus spp. & \\
Bush Baby Lima & $35.0 \mathrm{ab}$ \\
PI 642947 & $56.1 \mathrm{~cd}$ \\
PI 550212 & $56.2 \mathrm{~cd}$ \\
Bush Yellow Wax & $60.7 \mathrm{~cd}$ \\
PI 550213 & $61.4 \mathrm{~cd}$ \\
PI 549901 & $63.6 \mathrm{~d}$ \\
Soybean (G. max) & \\
DT97-4290 & $28.3 \mathrm{a}$ \\
Spencer & $42.5 \mathrm{bc}$ \\
Pharaoh & $78.2 \mathrm{~d}$ \\
Mean & 50.1 \\
\hline
\end{tabular}

${ }^{y}$ PI (plant introduction) accessions were obtained from the United States Department of Agriculture-Agricultural Research Service (USDA-ARS), National Plant Germplasm System (33). Bush Baby Lima and Bush Yellow Wax bean were purchased locally. DT97-4290 was obtained from USDA-ARS, Stoneville, MS; the other soybean genotypes were public releases from universities in the Midwest.

${ }^{\mathrm{z}}$ RAUDPC (relative area under disease progress curve) was calculated by dividing the area under disease progress curve by the number of days between the first and last date of necrosis measurement. Means followed by common letters were not significantly different by the least significant difference test at $P=0.05$. Coefficient of variation was $12.2 \%$. 
and CVs were lower in the greenhouse trials compared with the growth chamber trials. Though both environments are suitable, the greenhouse environment is often more available to researchers.

The cut-stem inoculation technique was not only shown to be useful in soybean $M$. phaseolina resistance evaluations but also may improve screening for $M$. phaseolina resistance in other crops, such as Phaseolus spp. Using the cut-stem technique in this study, M. phaseolina infection on Phaseolus spp. was similar to development of infection on soybean. RAUDPC on the $P$. lunatus genotype was comparable with DT97-4290, indicating that it may be a new source of resistance for edible bean crops. Additional comparisons are needed to determine whether the data from the cut-stem inoculation technique are correlated to results obtained in previous reports of evaluating Phaseolus spp. for charcoal rot resistance either in the field (33) or under controlled conditions (5).

Results from the aggressiveness experiments in this study showed that the cut-stem inoculation technique successfully distinguished differences in aggressiveness among soybean isolates of M. phaseolina, defined by relative amount of necrosis (RAUDPC) caused by each isolate. However, no significant interaction between isolate and genotype was found in this study, providing evidence for the lack of host specialization among soybean isolates of M. phaseolina toward soybean genotypes. The cut-stem technique could be used to study host specialization among $M$. phaseolina isolates collected from other host plants $(14,34)$, such as sorghum (7), common bean (19), and sunflower (1).

Although other techniques to evaluate plants for M. phaseolina resistance under controlled or semi-controlled environments $(3,17,35)$ have been reported, a standard procedure has not been widely adopted to screen for M. phaseolina resistance in soybean. Lack of standardized resistance evaluation practices limits comparison of results produced in different laboratories. In our trials, the cut-stem inoculation technique, which has several advantages over field tests, successfully distinguished relative differences among soybean genotypes for resistance to $M$. phaseolina. The most- and least-resistant genotypes were consistently distinguished with this technique. Differences among genotypes with intermediate colonization levels were subtle, and as a group, behaved similarly in response to $M$. phaseolina infection. The cut-stem technique could serve as a standard technique among researchers because of its ease of use and multiple advantages over field-testing.

\section{Acknowledgments}

We thank the United Soybean Board for their support of this research; and T. Herman, J. C. Rupe, and K. Whiting for their editorial comments

\section{Literature Cited}

1. Aboshosha, S., Attaalia, S., El-Korany, A., and El-Argawy, E. 2007. Characterization of Macrophomina phaseolina isolates affecting sunflower growth in El-Behera governorate, Egypt. Int. J. Agric. Biol. 9:807-815.

2. Bowen, C., and Schapaugh, W. 1989. Relationships among charcoal rot infection, yield, and stability estimates in soybean blends. Crop Sci. 29:4246.

3. Bristow, W. E., and Wyllie, T. 1984. Reaction of soybean cultivars to Macrophomina phaseolina as seedlings in the growth chamber and field. Trans. Mo. Acad. Sci. 19:5-10.

4. Campbell, C. L., and Madden, L. V. 1990. Introduction to Plant Disease Epidemiology. John Wiley and Sons, New York.

5. De la Peña-Devesa, D., Hernández-Delgado, S., Cantú-Almaguer, M. A., Arroyo-Becerra, A. L., Villalobos-López, M. A., González-Prieto, J. M., and Mayek-Pérez, N. 2009. Methods for Macrophomina phaseolina inoculation in common beans. Annu. Rep. Bean Improv. Coop. 52:94-95.

6. Dhingra, O. D., and Sinclair, J. B. 1978. Biology and pathology of Macrophomina phaseolina. Universidade Federal de Viçosa, Viçosa, Brazil.

7. Diourte, M., Starr, J., Jeger, M., Stack, J., and Rosenow, D. 1995. Charcoal rot (Macrophomina phaseolina) resistance and the effects of water-stress on disease development in sorghum. Plant Pathol. 44:196-202.

8. Doupnik, B. J. 1993. Soybean production and disease loss estimates for north central United States from 1989 to 1991. Plant Dis. 77:1170-1171.

9. Edmunds, L. 1964. Combined relation of plant maturity, temperature, and soil moisture to charcoal stalk rot development in grain sorghum. Phytopathology 54:513-517.

10. Fehr, W. R., Caviness, C. E., Burmood, D. T., and Pennington, J. S. 1971 Stage of development descriptions for soybeans, Glycine $\max$ (L.) Merr. Crop Sci. 11:929-931

11. Francl, L. J., Wyllie, T. D., and Rosenbrock, S. M. 1988. Influence of crop rotation on population density of Macrophomina phaseolina in soil infested with Heterodera glycines. Plant Dis. 72:760-764.

12. Ghaffar, A., Zentmyer, G. A., and Erwin, D. C. 1969. Effect of organic amendments on severity of Macrophomina phaseolina root rot of cotton. Phytopathology 59:1267-1269.

13. Hernández-Delgado, S. Reyes-Valdés, M. H. Rosales-Serna, R, and Mayek-Pérez, N. 2009. Molecular markers associated with resistance to Macrophomina phaseolina (Tassi) Goid. in common bean. J. Plant Pathol. 91:163-170.

14. Khan, S. N. 2007. Macrophomina phaseolina as causal agent for charcoal rot of sunflower. Mycopathologia 5:111-118.

15. Koenning, S. R., and Wrather, J. A. 2010. Suppression of soybean yield potential in the continental United States by Plant Diseases from 2006 to 2009. Plant Health Progress. Online publication. doi:10.1094/PHP-20101122-01-RS

16. Li, S., Hartman, G. L., and Boykin, D. L. 2010. Aggressiveness of Phomopsis longicolla and other Phomopsis spp. on soybean. Plant Dis. 94:10351040 .

17. Ma, J., Hill, C. B., and Hartman, G. L. 2010. Production of Macrophomina phaseolina conidia by multiple soybean isolates in culture. Plant Dis. 94:1088-1092.

18. Madden, L. V., Hughes, G., and van den Bosch, F. 2007. The Study of Plant Disease Epidemics. American Phytopathological Society, St. Paul, MN

19. Mayek-Pérez, N., C. , López-Castañeda, C., M. González-Chavira, R. García-Espinosa, J. Acosta-Gallegos, Vega, O. M. d. 1., and Simpson, J. 2001. Variability of Mexican isolates of Macrophomina phaseolina based on pathogenesis and AFLP genotype. Physiol. Mol. Plant Pathol. 59:257264.

20. Mengistu, A., Arelli, P., Bond, J. P., Shannon, J. G., Wrather, J. A., Rupe, J. C., Chen, P., Little, C., Canaday, C., Newman, M., and Pantalone, V. 2011 Evaluation of soybean genotypes for resistance to charcoal rot. Plant Health Progress. Online publication. doi:10.1094/PHP-2010-0926-01-RS

21. Mengistu, A., Ray, J. D., Smth, G. D., and Paris, R. L. 2007. Charcoal rot disease assessment of soybean genotypes using colony-forming units index. Crop Sci. 47:2453-2461.

22. Mengistu, A., Smth, J., Ray, J. D., and Bellaloui, N. 2011. Seasonal progress of charcoal rot and its impact on soybean productivity. Plant Dis. 95:1159-1166.

23. Mihail, J. D., and Alcorn, S. M. 1987. Macrophomina phaseolina: spatial patterns in a cultivated soil and sampling strategies. Phytopathology 77:1126-1131.

24. Papavizas, G. Some factors affecting survival of sclerotia of Macrophornina phaseolina in soil. Soil Biochem. 9:341-345.

25. Paris, R. L., Mengistu, A., Tyler, J. M., and Smith, J. R. 2006. Registration of soybean germplasm line DT97-4290 with moderate resistance to charcoal rot. Crop Sci. 46:2324-2325.

26. Pearson, C. A. S., Schwenk, F. W., Crowe, F. J., and Kelley, K. 1984 Colonization of soybean roots by Macrophomina phaseolina. Plant Dis. 68:1086-1088.

27. Senthilkumar, M., Swarnalakshmi, K., Govindasamy, V., Lee, Y., and Annapurna, K. 2009. Biocontrol potential of soybean bacterial endophytes against charcoal rot fungus, Rhizoctonia bataticola. Curr. Microbiol. 58:288-293.

28. Short, G. E., Wyllie, T. D., and Ammon, V. 1978. Quantitative enumeration of Macrophomina phaseolina in soybean tissues. Phytopathology 68:736741.

29. Siddiqui, Z. A., and Mahmood, I. 1993. Biological control of Meloidogyne incognita race 3 and Macrophomina phaseolina by Paecilomyces lilacinus and Bacillus subtilis alone and in combination in chickpea. Fundam. Appl. Nematol. 16:215-218

30. Smith, G. S., and Carvil, O. N. 1997. Field screening of commercial and experimental soybean cultivars for their reaction to Macrophomina phaseolina. Plant Dis. 81:363-368

31. Smith, G. S., and Wyllie, T. D. 1999. Charcoal rot. Pages 29-31 in Compendium of Soybean Diseases. G. L. Hartman, J. B. Sinclair and J. C. Rupe, eds. American Phytopathological Society, St. Paul, MN.

32. Songa, W., and Hillocks, R. 1996. Charcoal rot in common bean with special reference to Kenya. Int. J. Pest Manage. 42:213-219.

33. Songa, W., Hillocks, R. J., Mwango'mbe, A. W., Buruchara, R., and Ronno, W. K. 1997. Screening common bean accessions for resistance to charcoa rot (Macrophomina phaseolina) in eastern Kenya. Exp. Agric. 33:459-468.

34. Su, G., Suh, S.-O., Schneider, R. W., and Russin, J. S. 2001. Host specialization in the charcoal rot fungus, Macrophomina phaseolina. Phytopathology 91:120-126.

35. Surrette, S. B., Meints, P. D., and Trevathan, L. E. 2006. Evaluation of two methods to infect soybean with Macrophomina phaseolina (Deuteromycota) under controlled environmental conditions J. Miss. Acad. Sci. 51:134 139. 
36. Todd, T. C. 1993. Soybean planting date and maturity effects on Heterodera glycines and Macrophomina phaseolina in southeastern Kansas. Suppl. J. Nematol. 25:731-737.

37. USDA-ARS. National Genetic Resources Program. Germplasm Resources Information Network (GRIN). Online Database. National Germplasm Resources Laboratory, Beltsville, MD. http://www.ars-grin.gov/cgi-bin/npgs/ acc/display.pl?1444837
38. Vuong, T. D., Hoffman, D. D., Diers, B. W., Miller, J. F., Steadman, J. R., and Hartman, G. L. 2004. Evaluation of soybean, dry bean, and sunflower for resistance to Sclerotinia sclerotiorum. Crop Sci. 44:777783.

39. Winkler, H. E., Hetrick, B. A. D., and Todd, T. C. 1994. Interactions of Heterodera glycines, Macrophomina phaseolina, and mycorrhizal fungi on soybean in Kansas. J. Nematol. 26:675-682. 